\title{
Nucleate boiling at the forced flow of binary non-azeotropic mixtures in horizontal tubes
}

\author{
N.N. Mezentseva ${ }^{1 a}$, I.V. Mezentsev ${ }^{1}$, and A.V. Meleshkin ${ }^{1}$ \\ ${ }_{1}^{1}$ Institute of Thermophysics Siberian Branch, Russian Academy of Sciences, Lavrentiev Ave. 1, Novosibirsk, \\ 630090, Russia
}

\begin{abstract}
Analysis of experimental values of heat transfer coefficients obtained through investigation of nucleate boiling of the two-component non-azeotropic mixtures inside the horizontal smooth tubes by various authors is presented. In the zone of nucleate boiling, the experimental data are in good agreement with the calculation dependence.
\end{abstract}

\section{Introduction}

The non-azeotropic refrigerants are often used as the working medium in heat pumps [1-3]. The design and modeling of heat exchangers require the equations to calculate the heat transfer coefficient at boiling of non-azeotropic mixtures, which take into account peculiarities of the process. It is believed that during binary mixtures boiling, a low-boiling component is intensively released, and accordingly, the solution concentration changes, and this leads to a change in the wall-liquid temperature difference. At a change in solution concentration, the additional diffusion resistance is generated in liquid. Nonisothermality of phase transition or temperature glide also affects heat transfer. For the single-component substances, a significant number of correlations were proposed. However, only a few equations are suitable for refrigerant mixtures. The applicability of these general relations for mixtures should be proved.

The dependences presented in literature for calculation of heat transfer coefficients can be divided into two categories.

The models of Liu and Winterton (1991), Wattelet et al. (1994), Bivens and Yokozeki (1994), Shurshev (2006), Danilova (1983), etc. relate to the first category. In these studies, the calculation of heat transfer at liquid boiling in the horizontal tubes takes into account two components: heat transfer at the forced flow of pure liquid and heat transfer at nucleate boiling. In some works, it is proposed to summarize the contributions of evaporation and convection in the whole range of parameters, and other studies suggest using the asymptotic approach of Kutateladze (1961).

The second category engages investigations of Mishra et al. (1981), Chen (1966), Bennett-Chen (1980), etc. These works, like the studies of the first category, take into account two heat transfer components, but the effect of boiling is considered by means of the Lockhart-Martinelli parameter. The studies of Sami et al. (1992), Shin et al. (1996) take into account the difference of component concentrations in the liquid and vapor phases.

a Corresponding author: mnn@itp.nsc.ru 
Despite numerous empirical relationships, today there are no reliable and physically reasonable methodologies for calculating heat transfer at boiling of the non-azeotropic binary mixtures.

\section{Determination of heat transfer coefficient at nucleate boiling in horizontal tubes}

The heat transfer coefficient depends on many factors: flow regime, vapor mass flow ratio $\mathrm{x}$, mass velocity, pressure, heat flux, thermal-physical properties of liquid, tube diameter and length.

We have examined the experimental works of various authors on boiling the non-azeotropic mixtures R22/R142b and R32/R134a: Kim (2000), Shin (1997), Yoshida (1994), Zhang (1997), Shurshev (1997). The studies were carried out on the horizontal stainless steel and copper tubes; mass velocities were varied within $50-583 \mathrm{~kg} / \mathrm{m}^{2} \cdot \mathrm{s}$, specific heat flux was changed from 1 to $45 \mathrm{~kW} / \mathrm{m}^{2}$. The experimental results of Shin (1997) and Hihara (1989) on boiling the single-component refrigerants R32, R134a and R22 were treated to compare with the results obtained for the nonazeotropic mixtures. When processing the experimental data, the properties of single-component refrigerants and non-azeotropic mixtures were determined by the international database REFPROP Version 8.0 [4].

At boiling the refrigerants inside the horizontal tubes, three zones can be distinguished: convective motion of the single-phase liquid, nucleate boiling and evaporation at forced convection. The boundary between the zone of nucleate boiling and evaporation at forced convection can be determined by means of dependence $\mathrm{Bo} \cdot \mathrm{X}_{\mathrm{tt}}=1,5 \cdot 10^{-4}$. The detailed description is presented in [5].

It is known that at developed nucleate boiling in tubes, the heat transfer coefficient is described by the same dependences as at pool boiling [6]. The heat transfer coefficients were calculated for the zone of nucleate boiling of the single-component and non-azeotropic mixtures by dependences of Kutateladze (1961) [7], Cooper (1984) [8], and Gogonin (2006) [9]. The heat transfer coefficient, calculated by the Gogonin's dependence, which takes into account the effect of thermal-physical properties of the wall and its roughness on heat transfer, coincides well with the experimental data (Fig.1).

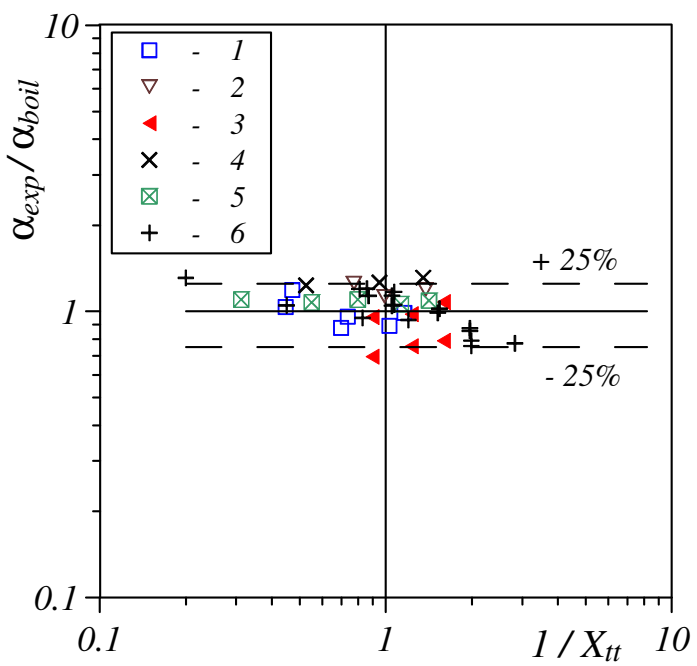

Figure 1. Change in the ratio of heat transfer coefficients depending on Lockhart-Martinelli parameter at nucleate boiling (1 - Shin R32/R134a, 2 - Kim R32/R134a, 3 - Yoshida R32/R134a, 4 - Zhang R32/R134a, 5 - Shin R134a, 6 - Shurshev R22/R142b). 
This dependence was based on processing the experimental data obtained at boiling of singlecomponent refrigerants, nitrogen, and propane:

$$
N u^{*}=0.01 \cdot \mathbf{R e}_{*}^{0.8} \cdot \mathbf{P r}^{1 / 3} \cdot b \cdot K_{t}^{0.4} \cdot \bar{R}_{z}^{0.2} \cdot\left(\frac{\lambda \cdot c \cdot \rho}{\lambda_{w} \cdot c_{w} \cdot \rho_{w}}\right)^{-0.2}
$$

The capillary constant is used as the characteristic dimension.

$$
N u^{*}=\frac{\alpha_{\text {boil }} \cdot \delta}{\lambda}
$$

\section{Conclusion}

To determine the heat transfer coefficients in the zone of nucleate boiling for both single-component refrigerants and non-azeotropic mixtures, we can recommend Gogonin's dependence. The deviation of experimental data from the results of calculation by this dependence does not exceed $\pm 25 \%$.

It was also determined that at intensive forced motion of non-azeotropic mixtures in tubes, the diffusion processes, in contrast to their pool boiling, do not play a significant role.

\section{Acknowledgments}

This work was financially supported by the Russian Foundation for Basic Research (grant № 14-0831621) and by the RF grant for state support of scientific researches performed under the direction of leading scientists (grant № 14.B25.31.0030, leading scientist Y. Kawazoe, Kutateladze Institute of Thermophysics, Siberian Branch, Russian Academy of Sciences)

\section{References}

1. Mezentseva N.N., Ogurechnikov L.A. Non-azeotropic mixtures in heat pumps // International Scientific Journal for Alternative Energy and Ecology; 2008, № 7, pp. 110-115.

2. Mezentseva N.N., Ogurechnikov L.A. Analysis of effective use of the mixtures of ozone-friendly coolants in vapor compression heat pumps under different operation conditions // Energetika i teplotekhnika. 2008, № 12, pp. 57-66.

3. Mezentseva N.N. Efficiency of vapor compression heat pumps based on non-azeotropic refrigerant mixtures // Thermophysics and Aeromechanics. 2011, Vol. 18, № 2, pp. 323-330.

4. NIST Thermodynamic and Transport Properties. Standard Reference Database 23, version 8.0. 2007.

5. Mezentseva N.N., Mukhin V.A., Mezentsev I.V. Boiling of single-component refrigerants and non-azeotropic mixtures inside the horizontal smooth tubes // Modern Science: researches, ideas, results, technologies. 2013. №1 (12), pp. 251-254.

6. Kutateladze S.S. The effect of circulation velocity on heat transfer coefficient at boiling in tubes // Energomashinostroenie. 1961. №1. p. 12.

7. Kutateladze S.S. Boiling heat transfer // International Journal of Heat and Mass Transfer, 1961, Vol. 4, pp. 31-15.

8. Cooper M.G. Saturation Nucleate Pool Boiling, A Simple Correlation, 1st U.K. National Conference on Heat Transfer, 1984, Vol. 2, pp. 785-793.

9. Gogonin I.I. The dependence of boiling heat transfer on the properties and geometric parameters of heat-transfer wall // High Temperature, 2006. Vol. 44, № 6, pp. 913-921. 